\title{
Germanica
}

\section{Nationalsozialistische Geschlechterideologie und Aktplastik im Dritten Reich}

L'idéologie sexuelle nationale-socialiste et le nu dans la statuaire allemande sous

le Troisième Reich

\section{Didier Herlem}

\section{OpenEdition}

\section{Journals}

Édition électronique

URL : http://journals.openedition.org/germanica/2213

DOI : $10.4000 /$ germanica. 2213

ISSN : 2107-0784

\section{Éditeur}

Université de Lille

\section{Édition imprimée}

Date de publication : 1 décembre 1994

Pagination : 79-89

ISSN : 0984-2632

Référence électronique

Didier Herlem, « Nationalsozialistische Geschlechterideologie und Aktplastik im Dritten Reich »,

Germanica [Online], 14 | 1994, Online erschienen am: 20 September 2013, abgerufen am 10 Dezember 2020. URL : http://journals.openedition.org/germanica/2213 ; DOI : https://doi.org/10.4000/ germanica.2213

Ce document a été généré automatiquement le 10 décembre 2020.

(c) Tous droits réservés 


\section{Nationalsozialistische Geschlechterideologie und Aktplastik im Dritten Reich}

L'idéologie sexuelle nationale-socialiste et le nu dans la statuaire allemande sous le Troisième Reich

Didier Herlem

1 In seiner Rede über «die deutsche Kunst als stolzeste Verteidigung des deutschen Volkes», die er am 1. September 1933 auf der Kulturtagung des NS-Parteitages hielt, bemühte sich Hitler, die Leitsätze nationalsozialistischer Kunstpolitik zu definieren. Was dabei herauskam, war sein Wunsch, «einen neuen Lebens-, Kultur- und Kunststil» entstehen zu sehen, der der «weltanschaulichen Erneuerung» und der «rassischen Klärung» der Zeit entsprechen würde. Die deutschen Künstler waren aufgefordert, sich zu mobilisieren, um die Verwirklichung der nationalsozialistischen Utopie zu fördern, um «die Fahnenträger der Zukunft» zu sein. Hitler sah wahrscheinlich ein, dass die damit verlangte «Gleichschaltung» der Kunst keine so leichte Sache sein würde, denn er gestand:

Ob die Vorsehung uns alle die Männer schenkt, die dem politischen Wollen unserer

Zeit und seinen Leistungen einen gleichwertigen kulturellen Ausdruck zu schenken vermögen, wissen wir nicht ${ }^{1}$.

2 Tatsächlich war die ästhetische Umsetzung desZeitgeschehens, die er wünschte, keine nachträglich reflektierende, sondern eine aktive, die sozusagen zum Zwecke hatte, das Geschehen selbst mitzugestalten oder gar zu bewirken.

3 Wie die deutschen Künstler auf diese Aufforderung Hitlers reagierten, soll nun anhand eines Beispiels veranschaulicht werden, nämlich, wie eine Komponente der NSWeltanschauung - die Geschlechterideologie - ihren ästhetischen Niederschlag in der Aktplastik des Dritten Reiches fand. 

insofern, als sie vom Rassenwahn, der sie kennzeichnete, untrennbar war. Schon 1926 hatte Parteiideologe Alfred Rosenberg in einem Artikel mit dem Titel «Mann und Weib» die nationalsozialistische Theorie auf diesem Gebiet festgelegt ${ }^{2}$. Jahrhunderts und 1942 in seinem Sammelband Blut und Ehre übernommen ${ }^{3}$. Man kann ihn daher als Referenz betrachten.

Diese Theorie geht von dem Prinzip der geschlechtlichen Polarität aus:

Innerhalb (der) Gegensätze der Rassen und Seelen schwingt das Leben aber noch ausserdem um zwei Pole: den männlichen und weiblichen ${ }^{4}$.

7

Die Spezifizität beider Pole wird, wie folgt, definiert:

Der Mann (ist) auf allen Gebieten der Forschung, Erfindung und Gestaltung dem Weibe überlegen, dessen Wert aber auf dem ebenso wichtigen, alles andere voraussetzenden Wert der Blutserhaltung und Rassenvermehrung beruht ${ }^{5}$.

Das Wesen der Frau wird durch «eine gewisse Fähigkeitslosigkeit» gekennzeichnet, die aber nur, betont Rosenberg, «die Folge des auf das Pflanzenhafte und auf das Subjektive gerichteten Wesens» ist. Der Mann geht daher an Welt und Leben «architektonisch», die Frau «lyrisch». Sie vertritt eine Welt

..., die in ihrer Schönheit und Eigenart der des Mannes nicht nach-, sondern ebenbürtig gegenübersteht ${ }^{6}$.

Demzufolge wäre es sinnlos, die beiden Geschlechter nivellieren, ausgleichen zu wollen. Mann und Frau «müssen als organische Wesen geachtet, in ihrer Eigenart gepflegt werden» ${ }^{7}$.

Diese wenigen Zitate genügen schon, um den Kern der NS-Geschlechterideologie zu begreifen. Sie beruht auf einer einmaligen Mischung von zwei Grundeinstellungen. Erstens wird eine klare Hierarchie zwischen Mann und Weib als Tatsache postuliert. Zweitens aber wird diese Hierarchie grundsätzlich verneint, und zwar im Namen einer radikalen Andersartigkeit der Geschlechter. Da beide eigentlich einander fremd sind, sollte jeder Vergleich, jedes Werturteil sinnlos sein.

11 Die Widersprüchlichkeit dieser Theorie erklärt sich dadurch, dass der Nationalsozialismus einerseits an den klassischen Anti-feminismus anknüpfte, wonach die Frau minderwertig sei, dass er aber andererseits, aufgrund seiner rassistischen und sozialdarwinistischen Auffassung des Lebens zwangsweise dazu geführt war, den biologischen Funktionen der Frau einen übergrossen Wert beizulegen. Die rhetorische Heiligung der Fortpflanzung und «Reinhaltung der Rasse» sollte vor allem die konkrete Diskriminierung der Frauen tarnen, ausgleichen, beschönigen. Denn ihnen war damit ein Tätigkeitsfeld zugewiesen, der einem Ausschluss aus vielen Aktivitäten gleichkam.

Dass eine solche Theorie nicht gerade leicht in die Praxis umzusetzen sein würde, wussten die Nazis sehr wohl. Seit der Industrialisierung Deutschlands war die Wandlung von Frauenstatus, -rolle und -aufgäbe im Vergleich zu ihrer Stellung in den 
alten patriarchalischen Gesellschaftsstrukturen beträchtlich gewesen. Die Entstehung einer Emanzipationsbewegung, die Forderung nach Gleichberechtigung beider Geschlechter auf allen Ebenen - Erziehung, Recht, Beruf, Politik, Sexualität - , das Schockerlebnis des Ersten Weltkrieges, wo Frauen Männer massiv ersetzt hatten, die partielle Erfüllung der Gleichheitsforderungen nach der Novemberrevolution, und nicht zuletzt der plötzliche Ausbruch der Modernität im Weimarer Staat, hatten die Mentalitäten beider Geschlechter stark beeinflusst. Ganz besonders die sexuelle Befreiung, das «Sich-Ausleben-Wollen», die freie Wahl des Partners, die Ablehnung der Mutterschaft durch Schwangerschaftsunterbrechung und Empfängnisverhütung, kurz, was man damals «die sexuelle Revolution» nannte, stimmten wenig mit den Grundlagen der NS-Geschlechterideologie überein.

Für die Nazis gehörte diese Entwicklung zu den widerlichsten Symptome des deutschen Verfalls. Der Bruch mit dem ewigen Gesetz der Polarität von Mann und Frau sowie die sexuelle Freiheit («die freie Liebe») haben, laut Rosenberg, zum «Rassen-und Geschlechterchaos» geführt. Daher seine Feststellung:

Wehrt sich niemand mehr gegen das Rassen- und Geschlechterchaos, dann ist der

Untergang unvermeidlich geworden ${ }^{8}$.

Was das Wehrprogramm Rosenbergs, nämlich die «Emanzipation der Frau von der Frauenemanzipation», konkret bedeuten sollte, erfuhren die Deutschen gleich nach der Machtübernahme. Die Frauenorganisationen wurden verboten und aufgelöst, der Kampf gegen die feministischen Ideen eingesetzt, die meisten Errungenschaften der Frauenbewegung seit ihrem Entstehen im Jahre 1863 wurden rückgängig gemacht oder aufgehoben. Die Frauen wurden aus den Parlamenten, aus den Führungsposten verdrängt und durch Männer ersetzt, verheiratete Beamtinnen im Staat- und Schulwesen entlassen; die Zahl der Studentinnen wurde drastisch reduziert, die gemeinsame Erziehung beider Geschlechter verboten, die Mädchengymnasien abgeschafft, der Zugang zu juristischen, richterlichen und ärztlichen Berufen den Frauen versperrt. Auf diese Weise wurde die Polarität der Geschlechter wiederhergestellt.

Gleichzeitig fing das neue Regime an, das «Rassen- und Geschlechtschaos» zu bekämpfen. Eine Fülle von eugenischen und diskriminierenden Massnahmen wurde getroffen: Zwangssterilisationen, Kontrolle der Eheschliessungen, EuthanasieProgramm «Aktion Gnadentod», Verfolgung deutscher Bürger «nicht arischer Abstammung», Verfolgung der sog. «Rasseschänder» und «Judensäue», der «Mischehen» und "Mischlinge», sowie der Homosexuellen, Massenvernichtungen in Rahmen der sog. «Endlösung der Judenfrage». Diese Massnahmen stellen den repressivzerstörerischen, den Tabula rasa-Aspekt dieser Politik dar.

Der totalitäre Anspruch der NS-Weltanschauung konnte sich aber nicht mit rein repressiven Massnahmen zufrieden geben. Das Ziel der Nazis schloss die Bekehrung des «gesunden Volkskörpers» zu ihrer Lebens- und Weltauffassung ein. Dafür wurden alle zu Verfügung stehenden Mittel eingesetzt, um Denkkategorien, Verhalten und sogar physische Erscheinung der einzelnen nach nazistischem Muster umzuformen. Propaganda und Massenorganisationen, Funk, Film und Schrifttum wurden restlos mobilgemacht. Dass in diesem riesigen Bekehrungsunter nehmen die bildenden Künste eine Rolle spielen konnten, war den Nazis von vorneherein klar. 

notwendig. Wie aus der anfangs zitierten Rede Hitlers hervorgeht, wünschte der Diktator das Entstehen eines neuen Kunststils, um die NS-Revolution symbolisch auszudrücken. Ferner war ihm die Gleichschaltung der Kunst notwendig, um die totale Homogenität des Volkskörpers zu beweisen: die Künstler durften keine Aussenseiter sein. Das Wichtigste war vielleicht aber, die emblematische Funktion der Kunst zur Durchsetzung seiner Weltanschauung auszunutzen.

Dass dabei die Plastik - und ganz besonders die Aktplastik - eine entscheidende Rolle spielen konnte, war nicht zu leugnen. Was konnte nämlich besser als eine Aktplastik das rassische Ideal der Nazis vorbildhaft verkörpern? Hinzu kam, dass gerade dieses Schönheitsideal an den griechischen Helden als Inbegriff des nordischen Menschen anknüpfte: die Aktplastik konnte damit den sozialdarwinistischen Rassenwahnsinn des Nazismus ästhetisieren, normalisieren, legitimieren und, warum nicht, veredeln. Die Versuchung, die Aktplastik verführerisch, etwa wie ein als Kunst objekt getarntes Werbematerial anzuwenden, war gross. Gerade auf dem Gebiet der NSGeschlechterideologie konnte die dreidimensionale Darstellung von nackten Körpern Prototypen liefern, die ohne Wort, allein durch unmittelbare Wahrnehmung, das Verhalten des Zuschauers beeinflussen. Der Akt sollte also nicht nur eine ästhetischdekorative Funktion erfüllen: auch seine ursprüngliche kultische Funktion galt es, Wieden aufleben zu lassen, um die Rasse und die heroischen Zeiten der NS-Revolution zu verherrlichen.

Dafür brauchte man aber willige Künstler. Waren die deutschen Bildhauer 1933 bereit, diese Aufgabe zu übernehmen? Anders als in der Malerei, wo die Säuberungsaktion «Entartete Kunst» auf einmal verheerende Folgen gehabt hatte, war die Bildhauerei wenig getroffen worden. Barlach, Belling, Otto Freundlich, Lehmbruck waren zwar als entartet eingestuft worden, aber die meisten deutschen Künstler setzten die klassische Tendenz fort, die Adolf von Hildebrand (1847-1921) von Maillol übernommen hatte. Diese Tendenz, die von Künstlern wie Kolbe, Klimsch, Scheibe, Bleeker und Albiker vertreten war, konnte den Nazis nur genehm sein. Sie war weder «formzerstörerisch» noch «formauflösend», weder «entartet» noch «primitiv». Diese Bildhauer bemühten sich, die menschliche Figur zu erhalten, die Würde des Menschen im Geiste der Aufklärung darzustellen. Der Nationalsozialismus schloss sich also an diese Kunstrichtung an, die sie als Vorstufe einer neuen, «zeitnahen» Plastik annahmen. Wie die meisten Plastiker auf die Aufforderungen Hitlers reagierten, erzählt der nationalsozialistische Architektur- und Kunstideologe Werner Rittich 1941 in der Zeitschrift Kunst im Deutschen Reich. Er stellt mit Bedauern fest

..., dass man sich in den Jahren nach der Machtübernahme auf das «Handwerkliche»

zurückzog und sich zunächst oft auch damit beschied,

und etwas weiter

..., dass in den letzten Jahren und AUCH JETZT NOCH ein grosser Teil der Bildhauer im wesentlichen nur wenig über die Erfüllung der handwerklichen Forderungen hinausgehen'.

Mit anderen Worten machten die meisten Plastiker nicht mit. Oder nur zurückhaltend. Von 1937 an wird jedoch in ihrer Aktproduktion eine gewisse Evolution spürbar. Der 
Stil wird glatter; impressionistische Überbleibsel werden zurückgenommen; ein neues Pathos wird hauptsächlich bei Kolbe vernehmbar. Aber ihre Plastiken bleiben im ganzen beim Menschenmass, was die NS-ErWartungen nur enttäuschen kann. Eine Bildhauerkunst, «die in Haltung und Stil das Leben- und Weltgefühl des deutschen Menschen unserer Zeit ausstrahlt oder sogar in gesteigerter Form gestaltet», Hess auf sich warten.

Zum Glück gibt es jedoch einige Ausnahmen, Plastiker, die auf dem Wege sind, «zu einem Stil zu kommen, der der adäquate Ausdruck unseres heutigen Denkens, Fühlens und Handelns ist». Der Wunderkünstler ist Arno Breker, der genau das tut, was das Regime verlangt. Breker, erklärt Werner Rittich, wagt sich ins «Überlebensgrosse». Durch die sichere Wahl des Themas zur Verkörperung eines umfassenden Geschehens eröffnet er

... zeitnahe, ja politische Ausblicke, obwohl das Thema zeitlos umfassend gestaltet ist. ${ }^{10}$

Mit diesen beiden Adjektiven: zeitnah, zeitlos, ist (fast) alles gesagt. Immer wieder erwartet der Nationalsozialismus von der Aktplastik, dass «sie zeitnahe, zeitbewegende Themen symbolisch und monumental gestaltet», indem sie das Abweichende, Monströse, Einmalige dieser Thematik durch ihre zeitlose Tarnung banalisiert. Was entscheidend erschien, war der Schritt «zur geistigen Überhöhung des Realen», der das Reale vergessen Hess. Erst im Juni 1943 konnte Werner Rittich mit Freude das Auftauchen einer jüngeren Künstlergeneration begrüssen, Plastiker nämlich

..., die sich erst innerhalb dieses Zeitraums für den Künstlerberuf entschieden haben, nachdem sie schon durch die Schule der Hitler-Jugend und des Arbeitsdienstes gegangen waren, deren Haltung und Charakter also schon vom Zeitgefühl, von den Zeitereignissen und der neuen Weltanschauung geformt und gefestigt waren, bevor sie das Werkzeug zum erstenmal in die Hand nahmen ${ }^{11}$.

Die Aktplastik im Dritten Reich ist also gar nicht so homogen und weltanschauungstreu, wie sie auf dem ersten Blick erscheinen mag. Die Zahl der Künstler, die den Wünschen des Regimes rückhaltlos entgegenkamen, ist relativ gering. Viele Aktdarstellungen, vor allem weibliche, sind heute nicht gleich als nazistische Kunst einzustufen.

Trotz dieser Einschränkung kann jedoch behauptet werden, dass es dem Regime gelungen ist, eine ästhetische Umsetzung seiner Geschlechter idéologie zu bekommen.

Der männliche wie der weibliche Akt verkörpern junge Menschen im Fortpflanzungsalter. Sie sind wohlproportioniert und wohlgeformt. Die Erotik, die sie ausstrahlen, ist nie aggressiv. Die anatomischen Details - einschliesslich primäre und sekundäre Geschlechtsmerkmale - sind realistisch zur Schau gestellt. Das Weinblatt scheint hier unbekannt zu sein. Die Menschentypen, die uns zur Bewunderung angeboten werden, sind durch ihr sportlichgesundes Aussehen gekennzeichnet. Die Empfehlung Rosenbergs der Frau betreffend, wonach «für ihre körperliche Ertüchtigung (...) durch Rhythmik, Turnen, Sport die gleiche Sorgfalt anzuwenden (sei) wie für den Mann», findet hier ihren idealen Niederschlag ${ }^{12}$. Frauen und Männer verleihen aber oft den Eindruck einer funktionellen Schönheit, die sich entweder als 
solide, zuverlässige "Qualitätsware» gibt, oder eine katzen-hafte Animalität aufweist. Bedenkt man den rassistischen Kern der NS-Weltanschauung, so erscheinen sie unmittelbar als das, was sie zuerst sind: die Proklamation der ästhetischen und physischen Überlegenheit der nordischgermanischen Rasse den anderen minderwertigen oder degenerierten gegenüber. Zweitens liefern sie dem einzelnen Zuschauer ein idealisiertes Spiegelbild des deutschen Volkes, mit welchem es sich stolz identifizieren kann. Die perfekte Schönheit dieser Prototypen verleiht drittens der Rassenpolitik einen Sinn. Als Entwürfe des künftigen deutschen Idealtypus, wonach man strebt, rechtfertigen sie die chirurgischen Eingriffe, die den Volkskörper zur rassischen Reinheit und Schönheit führen sollen.

Aber Mann und Frau sind in der Aktplastik selten zusammen dargestellt. Und wenn es doch der Fall ist, dann ist oft die Frau nicht als Mensch, sondern als Allegorie (der Heimat, des Todes) eingesetzt. Nur Kolbes «Menschenpaar» und Thoraks «Zwei Menschen» drücken eine gewisse Gemeinsamkeit beider Geschlechter aus. Thoraks "Urteil des Paris», das damals grossen Erfolg hatte, ist ein weiteres interessantes Beispiel. Drei überle-bensgrosse Frauenfiguren mimen eine fiktive Entkleidung. Ihnen gegenüber steht der Mann, ein Athlet, der da ist, um seine Wahl zu treffen. Die Frauen rivalisieren durch Schönheit und Erotik um die Gunst des Mannes. Er ist der Überlegene, sie hängen willfährig von seiner Entscheidung ab. Dieses Werk symbolisiert meisterhaft den nazistischen Willen, jede Gleichberechtigung zwischen Mann und Frau - auch im Sexualleben - endgültig zu vernichten und das Vorrecht des Mannes wiederherzustellen. Ausserdem ist das Thema der Auslese mit der Idee der Züchtung einer besseren Rasse verbunden. Darüber hinaus lässt die Gegenüberstellung von drei Frauen und einem Mann eine Andeutung an eine mögliche Vielweiberei vermuten, die den Plänen des Regimes nicht fremd war.

Die Überzahl von einzelnen Aktplastiken ist kein Wunder, insofern als die Bildhauerei zum Ziele hatte, die Polarität der Geschlechter und ihre jeweiligen Funktionen symbolisch auszudrücken. Dass man sie getrennt darstellt, ist also durchaus logisch: "Wir empfinden es als natürlich», wenn die Welt des Mannes und die des Weibes "geschieden bleiben", hatte Hitler im Jahre 1934 gesagt ${ }^{13}$. Dadurch wird die Andersartigkeit, das Geschlechtsspezifische von Status, Rolle und Aufgabe gemäss der NS-Ideologie hervorgehoben.

Der Mann wird aktiv dargestellt. Sein Tätigkeitsfeld ist die weite Aussenwelt. Er steht breitbeinig fest auf dem Boden oder vorwärtsstürmend. Er ist herrisch aggressiv in Posen grösster körperlicher und willenhafter Anspannung. Ganz besonders bei Breker drückt das Gesicht bis zur Karikatur den Zorn, die verbissene Hartnäckigkeit des völkischen Schicksalskämpfers aus. Die plastisch überbetonte Muskulatur, die ans heutige Body-Building erinnert, der dicke und zu lange Hals, der kleine Schädel signalisieren den radikalen Bruch mit dem aufklärerisch-vernunftsorientierten Menschenideal zugunsten des Instinkts, des Animalischen. Sie unterstreichen auch die unermüdliche Zeugungskraft seiner Männlichkeit.

31 Die Frau wird passiv dargestellt, auf sich selbst zurückgezogen. Ihr Tätigkeitsfeld trägt sie als Gebärmaschine in sich. Ihre Welt ist die der Anpassung, der pflichtbewussten Hingegebenheit. Sie wird in labiler Haltung, «tänzerisch bewegt» ${ }^{14}$, kniend, oft in der Haltung des hochstrebenden Körpers mit ausgebreiteten Armen und nach hinten gesenktem Kopf (wie z.B. im Thoraks «Frauenakt») dargestellt. Die Posen typisch weiblichen Anmuts treten immer wieder auf. Das Erotische bleibt jedoch von jeder 
Laszivität fern. Die Frau muss den Mann anziehen, aber die angestrebte Sexualität ist keine primär rekreative, sondern eine reproduktive. All diese weiblichen Akte vermitteln denselben Eindruck des Wartens, der geduldigen Verfügbarkeit: lauter rassisch einwandfreie Dornröschen, die wachbefruchtet sein möchten und ihre weiblichen Reize passiv ausstrahlen lassen. Die Botschaft ist für beide Geschlechter klar. Auswahl und Initiative gehören dem Mann. Die Frau soll sich damit abfinden und sich der naturgegebenen Ordnung fügen. Der weibliche Akt im Dritten Reich ist demzufolge die emblematische Darstellung des vom Regime erwünschten Verhaltensmusters beider Geschlechter. Der Schönheitskult, der sie begleitet, sollte wahrscheinlich vergessen lassen, dass nun die Frau, als rein biologisches Wesen definiert, zum Bürger zweiten Rangs geworden war: «Das deutsche Mädchen ist Staatsangehörige und wird mit ihrer Verheiratung erst Bürgerin», hatte Hitler in Mein Kampf bestimmt ${ }^{15}$.

Dass die Aktplastik des Dritten Reiches heutzutage «aufgeblasen» aussehen kann, eine gewisse «Leere» vermittelt oder gar lächerlich ist, scheint normal zu sein. Denn wie die Reklame ist sie eine Werbekunst für eine Ware - die NS-Weltanschauung -, die uns fremd geworden ist. Als instrumentalisierte Kunst war sie vom historisch-politischen Zusammenhang so abhängig, dass sie heute zum Teil sinnlos ist. Diese Männer- und Frauenkörper zeugen aber von einer Epoche, wo der totalitäre Rassenwahnsinn vorherrschte. Vielleicht wäre es daher angebracht, diese verfallenen Götter einer absurden Religion nicht ganz zu vergessen. Denn der Sozialdarwinismus ist noch lange nicht gestorben.

\section{NOTES}

1. - Adolf Hitler, «Die deutsche Kunst als stolzeste Verteidigung des deutschen Volkes», in: Reden des Führers, hrsg. v. Erhard Klöss, München, 1967, S. 118.

2. - Alfred Rosenberg, «Mann und Weib», in: Der Weltkampf, 1926/4.

3. - Alfred Rosenberg, Der Mythus des 20. Jahrhunderts, München, 1930. Ders., Blut und Ehre, 2 Bd, München, 1942, Bd I, S. 220-24.

4. - Alfred Rosenberg, Der Mythus des 20. Jahhunderts, S. 482.

5. - Ebenda, S. 483.

6. - Alfred Rosenberg, Blut und Ehre, Bdl, S. 220.

7. - Ebenda, S. 224.

8. - Ebenda, S. 220.

9. - Die Kunst im Deutschen Reich, 5. Jahrgang, Folge 8/9, August/September 1941, S. 249 und 250.

10. - Ebenda, S. 259.

11. - Die Kunst im Deutschen Reich, 7. Jahrgang, Folge 8/9, Juni 1943, S. 124.

12. - Alfred Rosenberg, Blut und Ehre, Bd I, S. 223.

13. - Max Domarus, Hitler, Reden und Proklamationen 1932-1945, hrsg. v. R. Lö-wit, Wiesbaden, 1973, S. $450-51$.

14. - Georg Bussmann, «Plastik», in: Kunst im 3. Reich, Frankfurt/M., 979/1, S. 248.

15. - Adolf Hitler, Mein Kampf, München, 1939, 410./414. Auflage, S. 491. 


\section{RÉSUMÉS}

Die Instrumentalisierung der Kunst durch den Nationalsozialismus bietet ein interessantes Beispiel für die ästhetische Umsetzung des Zeitgeschehens an. Dem NS-Regime ging es nämlich darum, den normalen Prozess der künstlerischen Widerspiegelung der geschichtlichen Entwicklung umzukehren. Kunst sollte das Geschehen mitgestalten oder gar untrennbarer Bestandteil der revolutionären Dynamik werden. Wie diese Instrumentalisierung durchgeführt wurde - und mit welchem Erfolg, wird anhand eines Beispiels dargelegt, nämlich, die NSGeschlechterideologie und ihr künstlerischer Niederschlag in der Aktplastik des Dritten Reiches. Von einer Analyse dieser Ideologie ausgehend, welche notwendige Hierarchisierung und Fremdartigkeit der Geschlechter zugleich postulierte und den biologischen Funktionen der Frau einen neuen, rassisch orientierten und sozialdarwinistisch fundierten Inhalt gab, bemüht sich diese Untersuchung, die NS-Geschlechterpolitik konkret zu skizzieren: Eugenismus und diskriminierende Massnahmen sollten den vom «Rassen- und Geschlechterchaos» (A. Rosenberg) bedrohten deutschen Volkskörper vom sonst unvermeidlichen Untergang retten. Dann wird die Einbeziehung der bildenden Künste in dieses Rettungsprogramm geschildert. Dass diese nur mühsam erfolgte und erst 1943 als befriedigend abgeschlossen wurde, zeugt vom Widerstand der Künstler, die sich weigerten, «über die Erfüllung der handwerklichen Forderungen» hinauszugehen. Deshalb erscheint die während des Dritten Reiches produzierte Aktplastik wenig homogen, obwohl ein Teil dieser Produktion als echte nazistische Kunst eingestuft werden kann. Die darauf folgende Analyse dieser Werke als Träger und Vermittler der NSGeschlechterideologie führt zum Schluss, dass sie, wie jede andere Werbekunst übrigens, dermassen zeitgebunden sind, dass sie heute, aus ihrem historischen Zusammenhang herausgerissen, zum Teil sinnlos geworden sind. Daher die Notwendigkeit, ihre ursprüngliche Funktion einer emblematischen Darstellung der vom Nationalsozialismus erwünschten Verhaltensmuster beider Geschlechter wieder ans Licht zu bringen.

L'instrumentalisation de l'art par le National-Socialisme offre un exemple intéressant de transposition esthétique de l'événement contemporain. Il s'agissait pour le régime d'inverser le processus normal de cette transposition. L'art devait non plus refléter, mais contribuer à créer l'événement, devenant ainsi un élément-clé de la dynamique révolutionnaire nazie. La mise en œuvre de cette instrumentalisation et ses résultats sont analysés et exposés à partir de l'idéologie sexuelle nationale-socialiste et de sa traduction artistique dans la statuaire du Troisième Reich. Partant de l'examen de cette idéologie qui postule à la fois la nécessaire hiérarchisation des sexes et leur irréductible spécificité, qui impose aux fonctions biologiques de la femme une finalité nouvelle fondée sur le racialisme et le darwinisme social, l'analyse prend en compte la traduction concrète de ces principes telle qu'elle s'est opérée dans la politique sexuelle nazie: eugénisme, exclusions et discriminations devaient sauver le peuple allemand menacé par « le chaos sexuel et racial » (A. Rosenberg) d'une déchéance sinon irrémédiable. Est alors abordé l'enrôlement des arts plastiques dans ce programme de sauvetage. Enrôlement qui ne fut guère aisé à obtenir, et que les responsables nazis eux-mêmes ne considérèrent comme réussi qu'à partir de 1943. Maints artistes rechignaient en effet à se transformer en propagandistes, à aller «au-delà de l'accomplissement des exigences artisanales ». Sans doute doit-on à cette résistance le caractère peu homogène des œuvres plastiques produites durant le Troisième Reich. Néanmoins, une partie de cette production peut être tenue pour "authentiquement nazie». L'analyse d'un échantillon de ces œuvres en tant que vecteurs de l'idéologie sexuelle nationale-socialiste conduit à la conclusion que, comme au demeurant tout art publicitaire ou de propagande, elles sont aujourd'hui, arrachées à leur contexte historique, partiellement privées de sens. D'où la 
nécessité de remettre en lumière leur fonction première, celle d'une mise en scène emblématique des modes de comportement que le National-Socialisme souhaitait faire adopter aux hommes et aux femmes du «Reich millénaire».

\section{AUTEUR}

\section{DIDIER HERLEM}

Université Charles-de-Gaulle - Lille III 